\title{
Modulating antioxidant activity and the controlled release capability of laccase mediated catechin grafting of chitosan
}

\author{
Suyeon Kim ${ }^{\mathrm{a}, *}$, Katherinne Isabel Requejo ${ }^{\mathrm{b}}$, Javier Nakamatsu ${ }^{\mathrm{b}}$, Karen N. Gonzales ${ }^{\mathrm{c}}$, \\ Fernando G. Torres ${ }^{\mathrm{c}}$, Artur Cavaco-Paulo ${ }^{\mathrm{d}}$ \\ a Engineering Department, Pontificia Universidad Católica del Perú PUCP, Av. Universitaria 1801, Lima 32, Lima, Peru \\ b Science Department, Pontificia Universidad Católica del Perú PUCP, Av. Universitaria 1801, Lima 32, Lima, Peru \\ c Department of Mechanical Engineering, Pontificia Universidad Católica del Perú PUCP, Av. Universitaria 1801, Lima 32, Lima, Peru \\ ${ }^{\mathrm{d}}$ Centre of Biological Engineering, Universidade do Minho, Campus de Gualtar, 4710-057 Braga, Portugal
}

\section{A R T I C L E I N F O}

\section{Article history:}

Received 14 July 2016

Received in revised form 11 October 2016

Accepted 7 December 2016

Available online 7 December 2016

\section{Keywords:}

Antioxidant activity

Controlled release

Laccase

o-quinones

Enzymatic grafting

\begin{abstract}
A B S T R A C T
Laccase from ascomycete Myceliophthora thermophila was used for the oxidation and grafting of catechin (CA) and chitosan (CS). The enzymatic grafting of CA into CS resulted in the improvement of natural properties of chitosan films. The successful grafting of CA to CS was supported by UV-vis and Fourier transform infrared (FT-IR) spectroscopies. The differences in the molecular weight and the degree of deacetylation of the CS affected on the enzymatic grafting rate and thus on the film properties like swelling behaviour, erosion rate, thermal property, tensile strength and breaking strain. The CS films prepared by enzymatic grafting presented high antioxidant activity compared to native CS films. The controlled release profiles of methylene blue and coomassie brilliant blue dye, having different acidity/basicity properties, loaded films were also studied with different release medium. The types of molecule loaded into films, the preparation manner of films and the release medium were decisive parameters affecting the release rate.
\end{abstract}

(c) 2016 Elsevier Ltd. All rights reserved.

\section{Introduction}

Biological polysaccharides are receiving a growing interest for the antioxidant and antimicrobial functionalisation of materials in food processing, treatment of fibres and medical/pharmaceutical applications [1-3]. Among the various polysaccharides, chitosan is currently the most studied for wound healing and controlled drug carrying materials with diverse forms such as films, beads, gels, scaffolds and nanoparticles. This is due to chitosan's unique properties of biodegradability, antibacterial activity, non-inflammatory property, non-toxicity, non-antigenic and high charge density [2-4]. Chitosan is composed of 2-amino-2-deoxy-D-glucose (with free amino groups) and 2-acetamido-2-deoxy-D-glucose (with acetylated amino groups) units linked through $\beta-(1 \rightarrow 4)$ bonds and it is obtained from the alkaline deacetylation of chitin. The

* Corresponding author at: Engineering Department, Pontificia Universidad Catolica del Peru, Av. Universitaria 1801, Lima 32, Peru.

E-mail addresses: skim@pucp.pe (S. Kim), kirequejo@gmail.com (K.I. Requejo), Javier.nakamatsu@pucp.pe (J. Nakamatsu), kareno3102@gmail.com (K.N. Gonzales), fernando.g.torres@gmail.com (F.G. Torres), artur@deb.uminho.pt (A. Cavaco-Paulo). properties of chitosan depend mostly on its molecular weight and degree of deacetylation (DDA) [5,6]. The deacetylation of chitosan is a process of hydrolysis of acetamide groups in chitin and normally conducted by severe heterogeneous alkaline treatments due to the resistance of such groups imposed by the trans arrangement of the C2-C3 substituent in the sugar ring [7]. The majority of the biological properties of chitosan is related to its cationic behaviour due to protonation of the amino groups in aqueous acidic solutions. Therefore, the DDA is the most important parameter on the biological activities of chitosan like biodegradability, biocompatibility, mucoadhesion, hemostatic, adsorption enhancer, anticholesterolemic, and antioxidant [6,7].

For wound treatment materials, the reduction potential of reactive oxygen is fundamentally necessary as much as an inhibition capacity to microorganism growth. The reactive oxygen that exists in the pathogenesis of wounds and injuries causes oxidative damages to proteins, nucleic acid, lipids as well as the depletion of mitochondrial DNA from human skin [8-10]. Chitosan has been reported as an effective antimicrobial agent showing high inhibition rate against to the microorganism growth [3]. Chitosan possess an antioxidant function but its activity level is very poor due to the lack of a $\mathrm{H}$-atom donor to serve as a good chain breaking antioxidant, especially in the case of medium and high molecular weights 
chitosan [11]. Flavonoids are well known natural phenolic antioxidants and are chemically applied to many substrates to improve their antioxidant activity $[10,12,13]$. The antioxidant capacity of flavonoids is very interesting and helpful for human health as they can act as anti-carcinogens, anti-inflammatory, and inhibitors of platelet aggregation in 'in vivo' and 'in vitro' studies [10]. In the presence of oxidative enzymes, flavonoids are the target of oxidation and form highly reactive o-quinones that can further couple with the free amino groups in chitosan [11,12,14,15].

Laccase (benzenediol: oxygen oxidoredcutase: EC 1.10.3.2) is one of the most promising oxidase enzymes used for biotechnological applications in a variety of industrial fields including pulp and paper, textile, food, biosensor, biofuel and pharmaceutical industries [16-18]. Laccase is able to catalyse the oxidation of aromatic substrates like ortho- and para- diphenols, aminophenols, polyphenols, polyamines, lignins and aryl diamines, as well as some inorganic ions coupled in the presence of molecular oxygen which is reduced to water during catalysis reaction [11]. In this research, we used laccase as an oxidative catalyst to modify chitosan films by grafting a strong antioxidant, catechin. Two types of chitosan with different degrees of deacetylation were used to verify the reaction degree of coupling between phenolic flavonoids and free amino groups in chitosan with laccase mediation. Chitosan films were further characterized by measuring tensile strength, thermal behaviour, swelling rate and antioxidant activity. Additional bioactive molecules were incorporated at chitosan films and their loading and release behaviours were studied.

\section{Experimental}

\subsection{Materials}

Two different chitosan compounds were studied for film formation. One was obtained from chitin (Paralomis granulosa exoskeleton) as described elsewhere with minor modifications $[19,20]$ and the other was a commercial product purchased from Sigma-Aldrich. The successive standard deacetylation procedures of chitin was performed in aqueous $50 \% \mathrm{NaOH}(\mathrm{w} / \mathrm{w})$ solution under nitrogen atmosphere, reflux and for $3 \mathrm{~h}$. Catechin and other chemical reagents were purchased from Sigma Aldrich and used without any further purification. Laccase from the ascomycete Myceliophthora thermophila (Novozym ${ }^{\circledR} 51,003$ ) was supplied by Novozymes Bagsvaerd, Denmark.

\subsection{Intrinsic viscosity determination}

Chitosan solutions of different concentrations were prepared in $0.2 \mathrm{M}$ acetic acid and $0.1 \mathrm{M}$ sodium acetate solution at $\mathrm{pH} 5$ and the viscosity measurement was conducted at $30^{\circ} \mathrm{C}$. The flow times of solvent $\left(t_{0}\right)$ and chitosan solutions $(t)$ were recorded and the specific viscosity $\left(\eta_{\mathrm{sp}}=\mathrm{t}-\mathrm{t}_{0} / \mathrm{t}_{0}\right)$ and the reduced viscosity $\left(\eta_{\text {red }}=\eta_{\mathrm{sp}} / \mathrm{c}\right)$ were obtained. The intrinsic viscosity $[\eta]$ was calculated graphically by extrapolating the curve of reduced viscosity versus concentration to zero concentration. The intrinsic viscosity corresponds to the intercept with y-axis obtained $[21,22]$.

\subsection{Determination of DDA and molecular weight of chitosan from NMR spectroscopy}

The DDA of chitosan was obtained from the $1 \mathrm{H}$ NMR spectrum, calculated from the relationship of the acetyl group hydrogen signal and the combined area of peaks corresponding to hydrogen in carbons 2-6 [23,24]. 1H NMR spectra were acquired on a Bruker $300 \mathrm{MHz}$ NMR AvanceIII 300 spectrometer with a Ultrashield 7.05 T superconductor magnet.
$11 \mathrm{mg}$ of chitosan was dissolved in $0.7 \mathrm{~mL}$ of $1 \% \mathrm{DCl}$ in deuterium oxide $\left(\mathrm{D}_{2} \mathrm{O}\right)$ and the experiments were run at $70^{\circ} \mathrm{C}$. 3-(Trimethylsilyl)propionic acid- $\mathrm{D}_{4}$ sodium salt was used as the reference and the deuteration degree was set a minimum $98 \%$ for the calibration of NMR spectra.

The molecular weight of chitosan was calculated by the Mark-Houwink-Sakurada (MHS) equation (Eq. (1)). The relationship between the molecular weight and viscosity are given below: where $[\eta]$ is the intrinsic viscosity, $M$ is the viscosity-average molecular weight, and $\mathrm{K}$ and $\alpha$ are the constants that depend on the DDA, the solvent and temperature. When $0.2 \mathrm{M}$ acetic acid/0.1 M sodium acetate solution ( $\mathrm{pH} 4.4$ ) is used as solvent and measurements are performed at $30^{\circ} \mathrm{C}, \mathrm{K}$ is $0.104 \times 10^{-3} \mathrm{~mL} / \mathrm{g}$ and $\alpha$ is 1.12 for DDA of $69 \%$ and $\mathrm{K}$ is $6.59 \times 10^{-3} \mathrm{~mL} / \mathrm{g}$ and $\alpha$ is 0.88 for DDA of over $90 \%$ [21].

$[\eta]=K M^{\alpha}$

\subsection{UV-vis spectrophotometer analysis}

Solutions of $2 \%(\mathrm{w} / \mathrm{v})$ chitosan compounds were prepared in a $1 \%(\mathrm{v} / \mathrm{v})$ acetic acid solution. The prepared solutions were further filtered with $0.8 \mu \mathrm{m}$ pore size of filter paper under air pressure. A $0.02 \mathrm{M}$ catechin solution was prepared in sodium acetate buffer ( $\mathrm{pH}$ 5). Chitosan and catechin solutions were firstly mixed to the same volume ratio and the final concentration of the solutions were set to $1 \%$ and $0.01 \mathrm{M}$, respectively. $167 \times 10^{-7}$ kat of laccase was added to initiate the enzymatic catalysis and the polymerisation of cetachin and the grafting of poly(catechin) into chitosan molecules were sequentially occurred in one bath process. To verify the rearrangement of molecules by enzymatic catalysis, UV-vis spectra were collected at room temperature before and after addition of laccase. The spectra were collected at certain time interval until reached $6 \mathrm{~h}$ of incubation time. The simply mixed solution of chitosan + catechin without laccase $(\mathrm{CS}+\mathrm{CA})$ was also analysed with spectroscopy and their spectra were compared with spectra of chitosan + catechin with laccase (CS + CA + LAC).

\subsection{Chitosan film formation}

Chitosan films were prepared by a casting/solvent evaporation technique [25-27]. Films were formed in Petri dishes ( $9 \mathrm{~cm}$ in diameter) with $20 \mathrm{~mL}$ of $1 \% \mathrm{CS}$ solutions then oven dried at $47^{\circ} \mathrm{C}$ for two days. Neutralization was carried out by immersion of dried films in a $1 \%(v / v) \mathrm{NH}_{3}$ solution for two hours. They were then rinsed with distilled water several times. The modification of CS was carried out by enzymatic grafting of polymerized CA into CS molecules using laccase as described in 2.4. After termination of laccase oxidation in $24 \mathrm{~h}$, the laccase and chitosan conjugates were removed from reaction solution by centrifugation as described in the literature [11]. A $20 \mathrm{~mL}$ of supernatant was taken and casted onto $9 \mathrm{~cm}$ diameter Petri dishes then oven dried at $47^{\circ} \mathrm{C}$ for two days. After neutralization of dried films, they were then rinsed with distilled water until no colour released.

\subsection{Characterizations of chitosan films}

\subsubsection{Thermal analysis}

The thermal behaviour of chitosan films was assessed using a 4000 Perkin-Elmer differential scanning calorimeter (DSC). Measurements were performed in two cycles, the first cycle was heated till $200{ }^{\circ} \mathrm{C}$ to remove all the evaporable compounds, then cooled till $30^{\circ} \mathrm{C}$. In the second cycle, the sample was heated from $30^{\circ} \mathrm{C}$ to $400^{\circ} \mathrm{C}$ with a heating rate of $3^{\circ} \mathrm{C} / \mathrm{min}$ under nitrogen gas passing at a flow rate of $20 \mathrm{~mL} / \mathrm{min}$. Samples weighted $8 \pm 0.5 \mathrm{mg}$. 


\subsubsection{Fourier transformed infrared (FT-IR) spectroscopy analysis}

A Perkin Elmer FT-IR 100 spectrophotometer was employed to record the IR spectra of samples i.e. CS, CS and catechin, and CS functionalised by enzymatic grafting of catechin The sample solutions were prepared then lyophilised. The $\mathrm{KBr}$ powder was used for background scanning. Same amount of $\mathrm{KBr}$ was mixed with lyophilised each sample for further analysis. The spectra were collected at a resolution of $8 \mathrm{~cm}^{-1}$, with 60 scans, over the range of $800-4000 \mathrm{~cm}^{-1}$ at room temperature. A background scan with no sample and no pressure was acquired before collecting the spectra of the samples.

\subsubsection{Swelling and degradation behaviours}

The swelling and degradation behaviours of chitosan films were studied in SIF (simulated intestine fluid without enzyme) and SGF (simulated gastric fluid without enzyme) which are commonly used for chitosan film characterization [27,28]. Films were cut $1.5 \mathrm{~cm} \times 1.5 \mathrm{~cm}$ pieces, then vacuum dried overnight before weighting $\left(W_{0}\right)$. Samples were submerged in $50 \mathrm{~mL}$ of medium and incubated in a shaking water bath at $37^{\circ} \mathrm{C}$. Samples were withdrawn from the medium at certain time intervals $(0,0.5,1,2,3,5$, 8 and $24 \mathrm{~h}$ ) and weighted $\left(W_{\mathrm{t}}\right)$ after removing the water from the surface of films with filter paper. The swelling rate was calculated following Eq. (2). The measurements were carried out in triplicate and the average values were determined

Swellingrate $(\%)=\frac{W_{t}-W_{0}}{W_{0}} \times 100$

Finally, after $24 \mathrm{~h}$ of incubation in medium, films were taken and again vacuum dried overnight to measure the erosion rate. The erosion rate of chitosan films was obtained by measuring the weight of films before and after incubation and presented as weight loss (\%) following the Eq. (3). All measurements were carried out in triplicate and their average values were determined.

Erosion $\operatorname{rate}(\%)=\left(W_{0}-\frac{W_{t}}{W_{0}}\right) \times 100$

\subsubsection{Tensile strength measurements}

The mechanical properties of chitosan films were determined by tensile tests. A bench top tensile testing machine (ESM Mark-10), equipped with a $100 \mathrm{~N}( \pm 0.5 \%)$ load cell was used, with a crosshead speed set at $10 \mathrm{~mm} / \mathrm{min}$. Three samples were tested per chitosan type, and each sample was cut into strips of $0.5 \mathrm{~cm} \times 3 \mathrm{~cm}$. These tests were performed at room temperature. Tensile strength, strain at break and Young's modulus values were recorded. Measurements were done in triplicate and average values and standard deviation are presented.

\subsubsection{Antioxidant activity measurement}

The antioxidant properties of chitosan films were evaluated according to a modified ABTS radical cation decolourization assay method $[10,29]$. ABTS $^{\circ+}$ free radical in solution was obtained from the reaction with potassium persulphate overnight in the dark at room temperature and then diluted with distilled water until the absorbance (Abs) reached $0.7 \pm 0.02$ at $734 \mathrm{~nm}$. Film samples of $10 \mathrm{mg}$ were immersed in $3 \mathrm{~mL}$ of diluted $\mathrm{ABTS}^{\circ+}$ free radical solution at room temperature and taken after 0, 30, 60 and $120 \mathrm{~min}$. The absorbance was measured at $734 \mathrm{~nm}$ and the scavenging ability of chitosan films was evaluated following Eq. (4). In Eq. (4), the Abs control is the absorbance values of diluted $\mathrm{ABTS}^{\circ}$ free radical solution without samples and Abs sample is the absorbance values of and diluted $\mathrm{ABTS}^{\circ+}$ free radical solution with chitosan films. The measurements were carried out in triplicate and the average values were determined.

Antioxidant activity $(\%)=\frac{\mathrm{Abs}_{\text {control }}-\mathrm{Abs}_{\text {sample }}}{\mathrm{Abs}_{\text {control }}} \times 100$
Table 1

DDA values obtained from ${ }^{1} \mathrm{H}$ NMR spectra and molecular weight determined from Mark-Houwink-Sakurada (MHS) equation of chitosan from Sigma-Aldrich and prepared by deacetylation of chitin provided by Idebio were presented.

\begin{tabular}{lll}
\hline & DDA (\%) & MW (kDa) \\
\hline $\begin{array}{l}\text { Chitosan from Sigma-Aldrich } \\
\text { chitosan obtained by deacetylation }\end{array}$ & 69 & 1370 \\
\hline
\end{tabular}

\subsection{6. 'In vitro' leaching behaviour}

To study the leaching behaviour of chitosan and modified chitosan films, methylene blue (MB) and coomassie brilliant blue (CB) dyes were employed as model molecules. $\mathrm{MB}$ and $\mathrm{CB}$ dyes are broadly used for biology, biochemistry and medicine applications. The MB dye is a basic dye which reacts with negatively charged compounds [30,31] and sulfonic acid groups in CB dye react with positively charged amino groups by ionic interactions [32]. They have different acidity/basicity properties in solution, MB becomes positively charged and CB negatively charged. $0.025 \mathrm{~g}$ of dyes were mixed to $50 \mathrm{~mL}$ of chitosan solutions ( $0.5 \mathrm{~g} \mathrm{~L}^{-1}$ dye conc.) and stirred for $3 \mathrm{~h}$. Chitosan solutions mixed with dyes were then employed to the preparation of chitosan films. The chitosan films containing $\mathrm{MB}$ and $\mathrm{CB}$ dyes were further tested to verify their leaching profiles in different buffer medium. $0.03 \mathrm{~g}$ was taken from each film of chitosan and modified chitosan containing dyes and then suspended in test tubes containing $50 \mathrm{~mL}$ of buffer medium. The incubation was performed using water bath at $37^{\circ} \mathrm{C}$ for $24 \mathrm{~h}$ under mild agitation. A $2 \mathrm{~mL}$ of the medium was withdrawn after certain time intervals and replaced with an equal volume of fresh medium. The release of molecules was quantitatively monitored by UV-vis spectroscopy in the range of $190-800 \mathrm{~nm}$. The buffer medium for leaching profiles of dyes from chitosan films were SIF and SGF. The maximum absorbance of dyes of $\mathrm{MB}$ was detected at $670 \mathrm{~nm}$ in both SIF and SGF solution. The maximum absorbance of $C B$ was obtained at $595 \mathrm{~nm}$ and $615 \mathrm{~nm}$ in SIF and SGF buffer solutions, respectively. The measurements were carried out in triplicate and the average values were determined.

\subsection{Statistic analysis}

Data was statistically analysed through $t$-test in excel and $p$ value $<0.05$ was considered as statistically significant. Data are presented as mean \pm standard deviation (SD) with samples number (n) $\square 3$.

\section{Results and discussion}

Chitosan is the N-deacetylated derivative of chitin and can be obtained by deacetylation of chitin. In this study, we prepared chitosan with high degree of deacetylation (DDA) and the properties for film formation were compared with commercial chitosan. DDA of two types of chitosan were identified by NMR spectroscopy analysis and the molecular weight (MW) was calculated by the Mark-Houwink-Sakurada (MHS) equation. Both values of DDA and MW were presented in Table 1 . The MW and DDA of chitosan are affecting their physiochemical and biological properties such as crystallinity, hydrophilicity, degradation and cell response so on [6,33]. Chitosan obtained by deacetylation (CS95) has higher rate of DDA and lower value of MW, $95 \%$ and $670 \mathrm{kDa}$, respectively, comparting to commercial chitosan (CS69) with 69\% and $1370 \mathrm{kDa}$, respectively.

The effects of the DDA and MW of CS, catechin (CA) presence, and laccase (LAC) catalysis on the viscosity were studied and the results obtained are presented in Table 2. Since the MW and DDA of CS are decisive factors on viscosity, the CS69 showed a higher viscosity than CS95. An increase in molecular weight and decrease 
Table 2

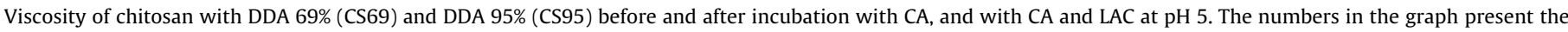
value of viscosity changes (\%)

\begin{tabular}{|c|c|c|c|c|c|c|}
\hline Samples & CS69 & $\mathrm{CS} 69+\mathrm{CA}$ & $\mathrm{CS} 69+\mathrm{CA}+\mathrm{Lac}$ & CS95 & $\mathrm{CS} 95+\mathrm{CA}$ & $\mathrm{CS} 95+\mathrm{CA}+\mathrm{Lac}$ \\
\hline Viscosity (cps) & 863 & 806 & 744 & 469 & 461 & 482 \\
\hline Increase of viscosity (\%) & Control & -7 & -14 & Control & -2 & 3 \\
\hline
\end{tabular}

Bold values show the degree of viscosity change after incubation with CA, and with CA + LAC.
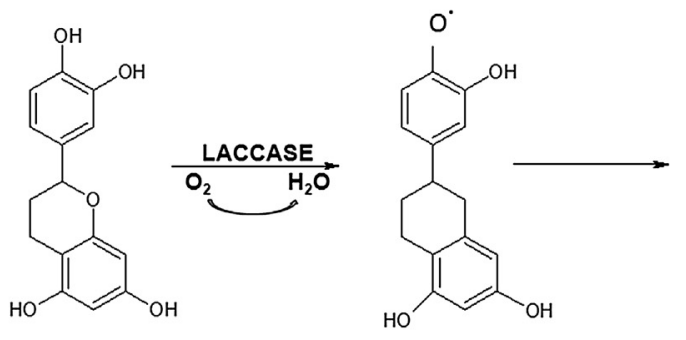<smiles>O=C1C=CC(C2CCc3c(O)cc(O)cc3C2)=CC1=O</smiles>

Catechin

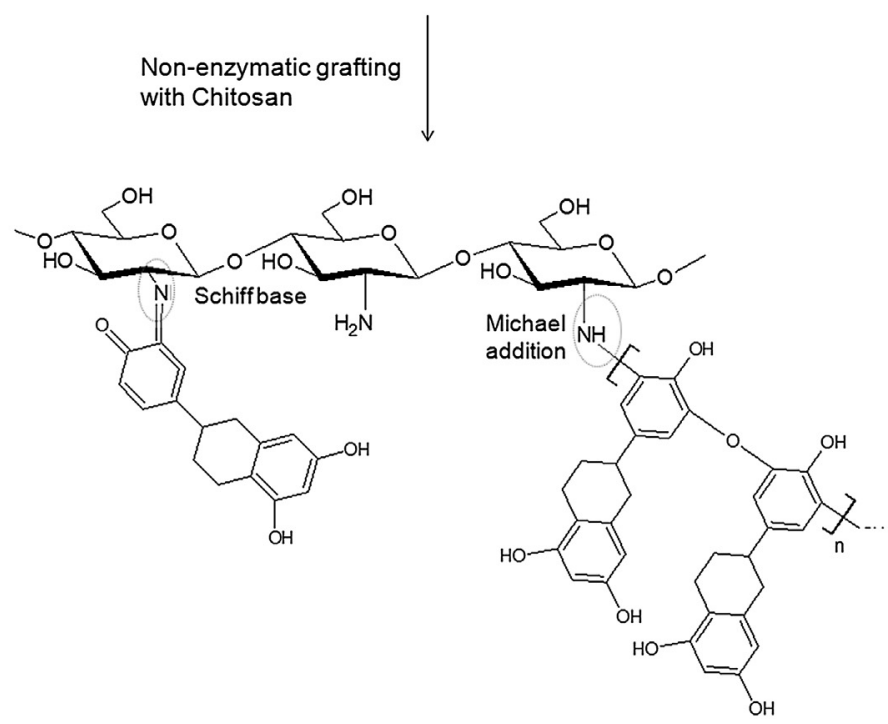

Fig. 1. Estimated schematic presentation of enzymatic oxidation of catechin by laccase and non-enzymatic grafting with chitosan.

in DDA cause low solubility and high viscosity due to entanglement of chains and low numbers of free amino groups [34]. The DDA of chitosan is directly related to the viscosity which plays an important role in the preparation of medical/pharmaceutical applications $[6,7,35]$. Compared to CS95, the viscosity of CS69 was influenced more by enzymatic CA grafting, showing higher rate of reduction $(-14 \%)$. The addition of CA and enzymatic catalysis decreased the viscosity in all samples except in the case of the incubation of CS95 with CA + LAC ( $3 \%$ increase). This could be explained by an increase of electrophoretic mobility by catechin conjugating into the structure of chitosan molecules [10]. The phenolic compounds form $o$-quinones which are highly reactive electrophilic compounds formed by a radical reaction and this radical reaction can occur naturally but can be accelerated by enzymatic catalysis. The reactive $o$-quinones formed are further covalently attached to nucleophilic amine groups in chitosan through Schiff-base and/or Michael-type addition reaction [36,37]. The expected mechanism is presented in Fig. 1. In the case of CS95 incubated with CA + LAC, the high rate of CA oligomers or polymers was substituted to the site of the hydrophilic and free amine groups in CS. The phenolic polymers with high molecular weight generated by enzymatic oxidation are less soluble than phenolic monomers or oligomers [38] and might provoke a slight increase in the viscosity of CS95 solu- tion since it has a higher number of free amino groups available to react with CA polymers than CS69.

In this study, laccase-mediated reactions of CA and CS were spectrophotometrically studied using a UV-vis spectrophotometer. The spectra were collected before and after laccase addition to each solution of CS69 and CS95 in the presence of catechin. The obtained results are presented in Fig. 2. After incubation with laccase, the mixed solutions of CS and CA turned dark brown producing an increase of band absorption in the visible area, which indicates a polymerisation or/and crosslinking [13,37,39]. A significant peak at $280 \mathrm{~nm}$ was detected in both CS69 + CA and CS95 + CA solutions and their intensity increased by addition of laccase. Resulting bands emerged at 380 and $450 \mathrm{~nm}$ and their intensity increased constantly with reaction time which is typically observed in the coupling reaction between reactive $o$-quinones and amino groups in a Michael addition reaction [37]. The rate increase of the peak intensity in the visible region with time was higher in CS95 than in CS69 solution. The difference of DDA and the number of free amino groups in CS might affect the reaction rate. The higher DDA means a higher amount of available amino groups to react with 0 quinone in oxidised CA. Most chemical and biological reactions of CS relate to its cationic character, therefore, it should be expected that CS95, which has a higher DDA, would interact more favourably with CA polymers. At the same time, the higher molecular weight 

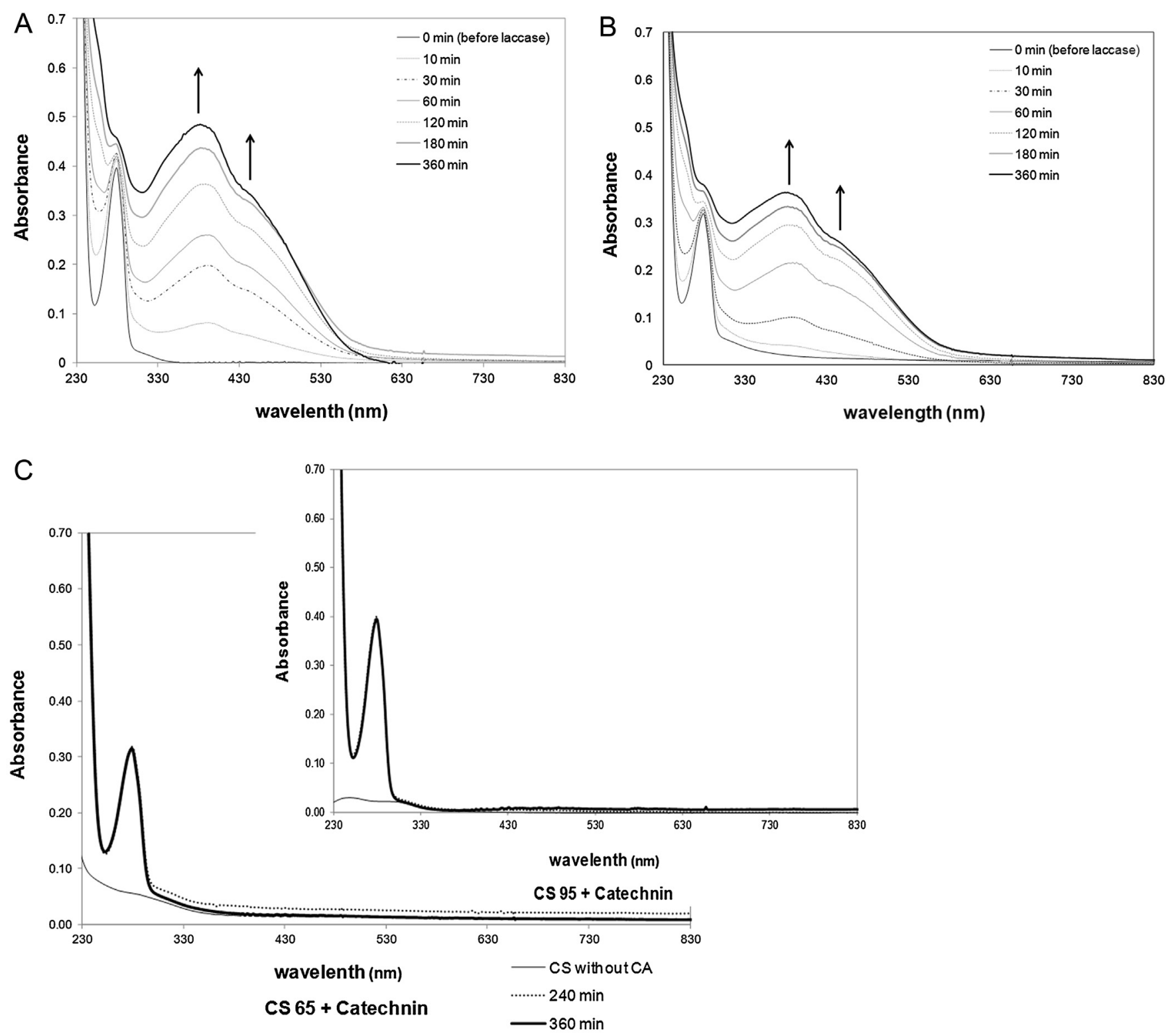

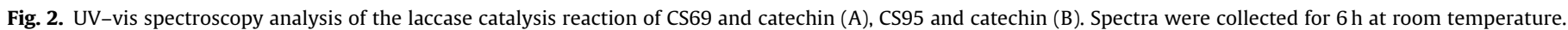
The spectra was also collected in the CS69 and CS95 solutions without laccase (C).

of CS65 might supplied higher structure hindrance to react with CA polymers than CS95. In a previous research, the high affinity of CA polymers obtained from laccase catalysis for cationic materials resulted in stronger colour absorbance onto cationised fibres than non-cationized fibres [10]. As controls, CS69+CA and CS95+CA solutions without laccase were also studied (Fig. 2C) and resulted in no peak appearance. The spectra were collected for $6 \mathrm{~h}$ but there were no notable changes in peak shape or intensity in both UV and visible area.

The thermo behaviour of native CS and modified CS films by enzymatic grafting was studied using DSC. The common peak, close to $88-90^{\circ} \mathrm{C}$, attributed by evaporation of absorbed water not detected due to pre heating cycle performing $\left(\sim 200^{\circ} \mathrm{C}\right)$. The thermograms showed an exothermic peak of CS films at around $280-290^{\circ} \mathrm{C}$ (Fig. 3 ). These peaks are accounted for by the decomposition of the amide group of chitosan $[40,41]$ and the peak appeared at higher temperature on CS $95(291.4 \pm 0.2)$ comparing to CS 69 $(281.7 \pm 0.4)$. This might be due to their different DDA level. The higher DDA and higher crystallinity of chitosan bring an increase of stability to molecules and it might result in an increase of degradation temperature [41]. In Fig. 3, the temperature of degradation slightly decreases when the blending CS with CA and an enzymatic grafting of CA to CS occurred. Comparing to CS95, the CS65 films were less affected by CA presence and enzymatic grafting to CA. The thermograms of CS films can be differed from those of physical mixture and chemical bonds compositions [42].

The catechin grafting onto CS95 by laccase catalysis was evidenced by FT-IR spectra. Fig. 4 shows the CS, CS + CA and CS functionalized by enzymatic grafting of $C A$. The spectrum of chitosan without any modification presents a strong absorption in the region $3200-3500 \mathrm{~cm}^{-1}$ and attributed to $\mathrm{NH}$-stretching [14]. The two specific peaks at $1670 \mathrm{~cm}^{-1}$ and $1580 \mathrm{~cm}^{-1}$ corresponding to amide I and amide II bands and a peak at $1380 \mathrm{~cm}^{-1}$ attributed to NH-bending of the glucosamine unit $[11,13]$. The characteristic peaks of CS saccharide structure were found at $1150 \mathrm{~cm}^{-1}$ (anti-symmetrical stretching of $\mathrm{C}-\mathrm{O}-\mathrm{C}$ bridge) and at $1080 \mathrm{~cm}^{-1}$ (skeletal vibration involving C-O stretching) [11,13,14]. The spectrum of CS functionalized by enzymatic grafting of CA shows the 


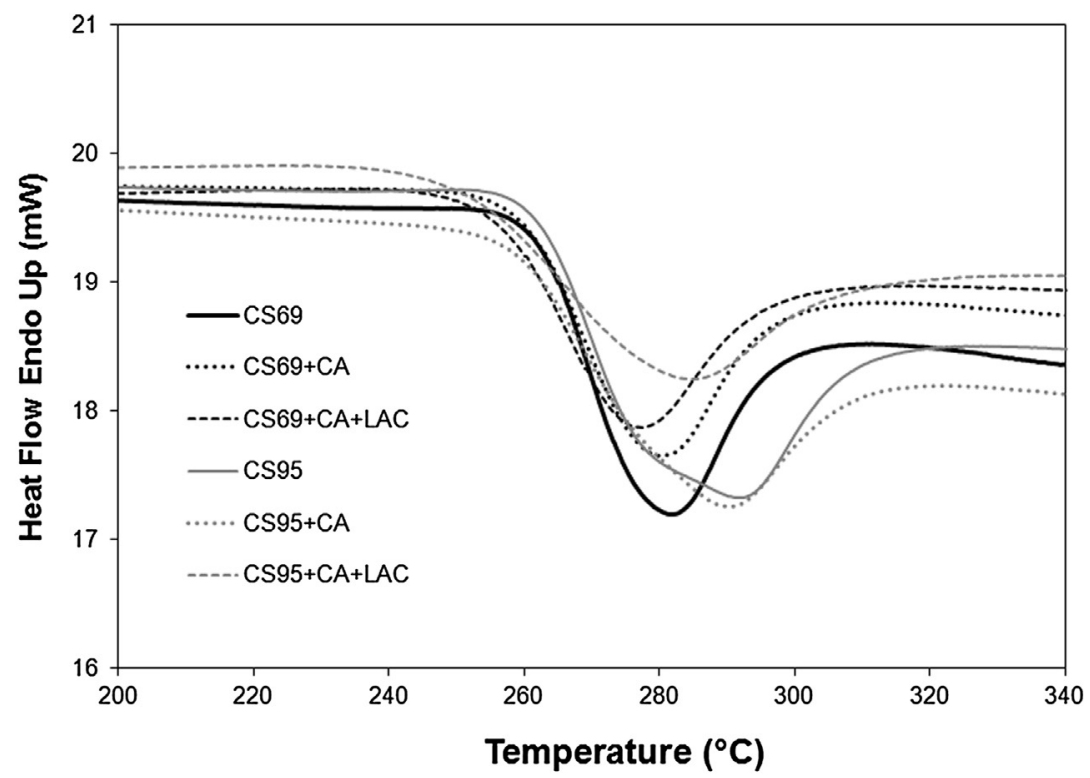

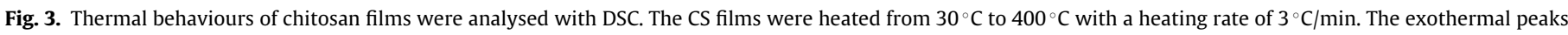

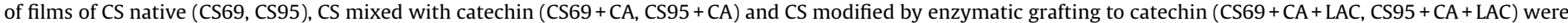
observed near $280-290^{\circ} \mathrm{C}$.

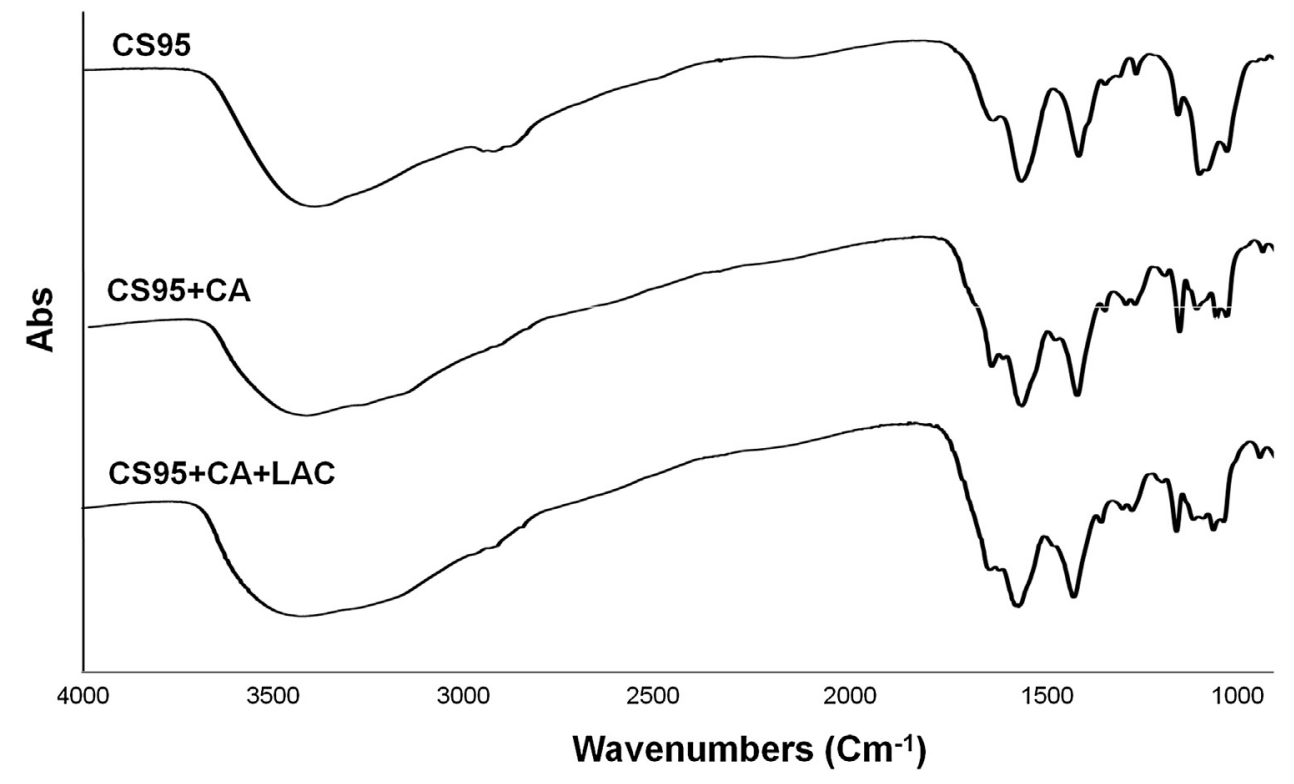

Fig. 4. FT-IR spectra of CS95, CS95 and catechin, and CS95 functionalized by enzymatic grafting of catechin. The spectra were showed from 1000 to 4000 cam ${ }^{-1}$.

Table 3

Data of weight, thickness, equilibrium water uptake (EWu, \%) and weight loss (\%) of CS films in SIF solution after $24 \mathrm{~h}$ at $37{ }^{\circ} \mathrm{C}$ is presented as mean \pm SD ( $\mathrm{n}=3$ ).

\begin{tabular}{|c|c|c|c|c|}
\hline Samples & Initial weight (mg) & Thickness $(\mu \mathrm{m})$ & $\mathrm{EWu}(\%)$ & Weight loss (\%) \\
\hline CS69 & $18.7 \pm 1.7$ & $47.5 \pm 0.7^{*}$ & $203.2 \pm 23.4$ & $-0.4 \pm 0.4$ \\
\hline $\mathrm{CS} 69+\mathrm{CA}$ & $13.7 \pm 1.7^{*}$ & $36.5 \pm 0.5^{*}$ & $201.5 \pm 18.3$ & $-3.0 \pm 0.8$ \\
\hline $\mathrm{CS} 69+\mathrm{CA}+\mathrm{Lac}$ & $16.7 \pm 1.3$ & $38.2 \pm 1.0^{*}$ & $160.5 \pm 8.9^{*}$ & $-0.8 \pm 1.1$ \\
\hline CS95 & $10.3 \pm 0.4$ & $31.5 \pm 2.8$ & $90.7 \pm 19.6$ & $\mathbf{0 . 0} \pm \mathbf{0 . 6}$ \\
\hline $\mathrm{CS} 95+\mathrm{CA}$ & $13.3 \pm 2.0^{*}$ & $36.0 \pm 1.2$ & $92.7 \pm 2.5$ & $-3.0 \pm 1.0$ \\
\hline $\mathrm{CS} 95+\mathrm{CA}+\mathrm{Lac}$ & $15.0 \pm 2.8^{*}$ & $38.8 \pm 2.0^{*}$ & $94.2 \pm 21.2$ & $-2.5 \pm 1.2$ \\
\hline
\end{tabular}

Bold values present the most different sample among all film samples (CS 95).

* Significantly different was $\mathrm{p}<0.05$ compared with non modified chitosan films.

bands that evidence the grafting of CS and CA, catalysed by the enzyme. The two adsorption bands are corresponding the phenolic oligomers or polymers and the first peak at $1023 \mathrm{~cm}^{-1}$ is evidenc- ing the extended polymerisation by showing the attribution to the $\mathrm{C}-\mathrm{O}$ stretching vibration and the second peak at about $1053 \mathrm{~cm}^{-1}$ contributing to the phenolic acids' $\mathrm{C}-\mathrm{C}$ linkages [11]. The specific 


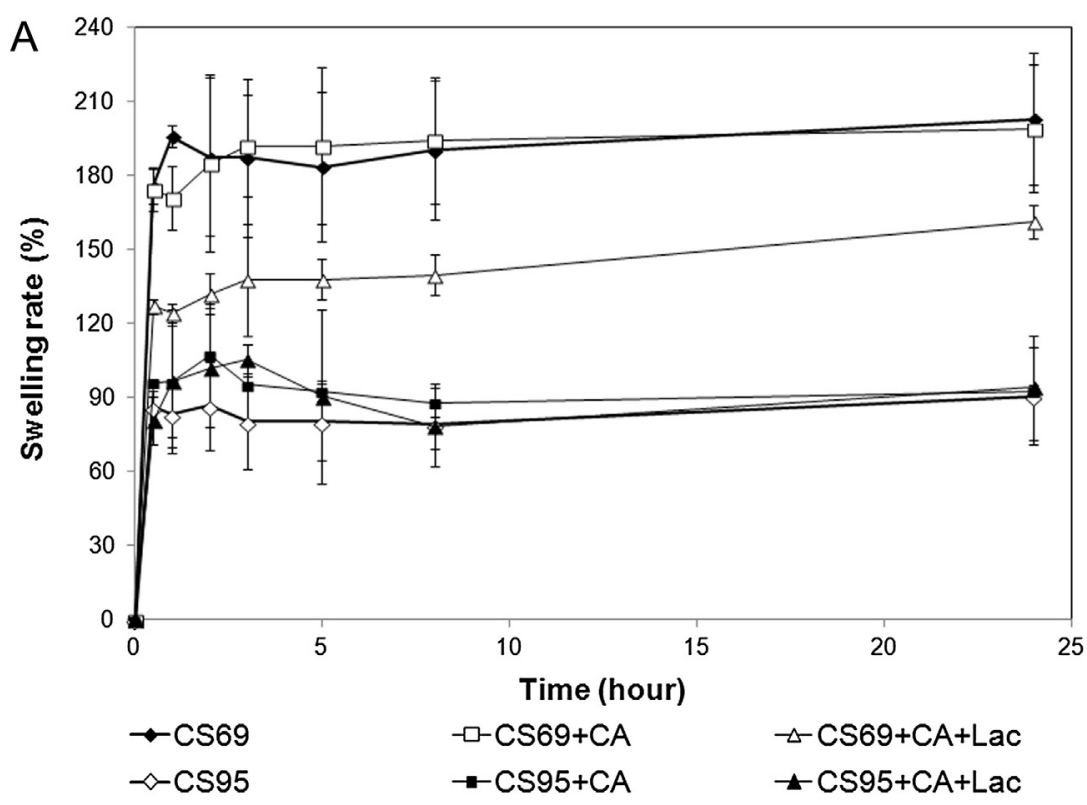

B

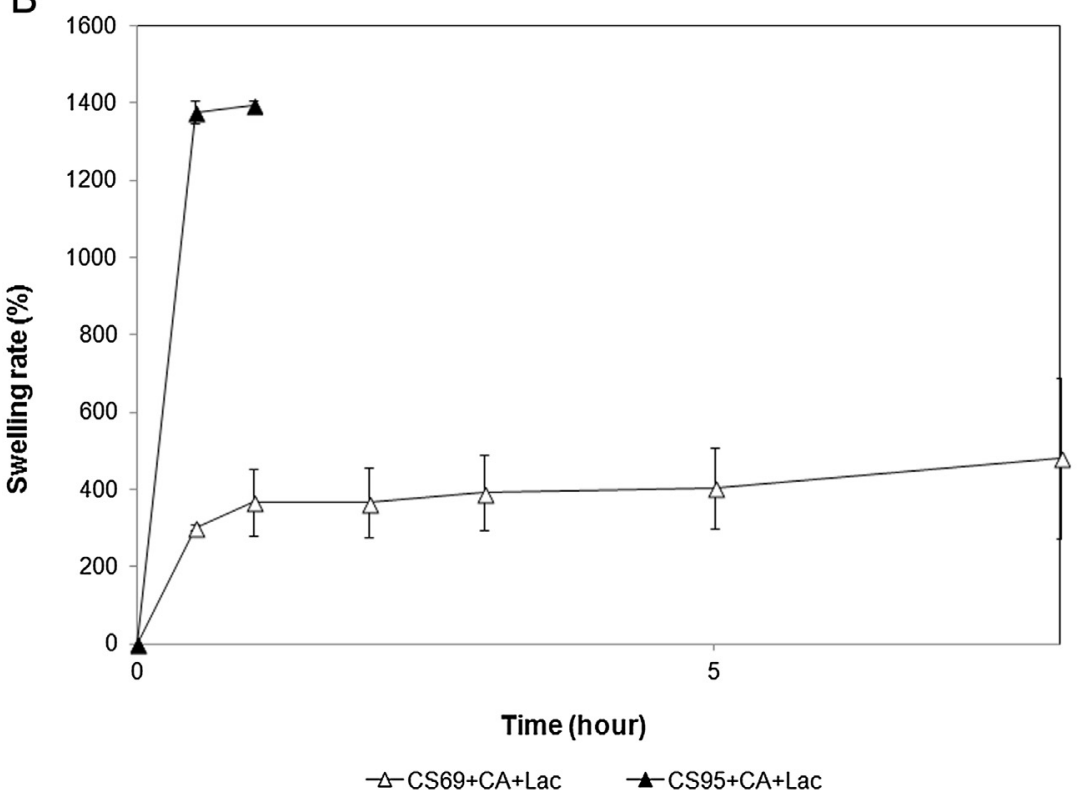

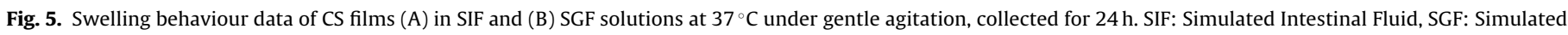
Gastric Fluid.

Table 4

Tensile strength (UTS) and elongation of CS, CS + CA, and CS + CA + LAC films.

\begin{tabular}{|c|c|c|c|c|c|c|}
\hline Samples & CS69 & $\mathrm{CS} 69+\mathrm{CA}$ & $\mathrm{CS} 69+\mathrm{CA}+\mathrm{Lac}$ & CS95 & $\mathrm{CS} 95+\mathrm{CA}$ & $\mathrm{CS} 95+\mathrm{CA}+\mathrm{Lac}$ \\
\hline UTS (MPa) & $\mathbf{5 7 . 0} \pm \mathbf{0 . 9}$ & $33.2 \pm 1.6$ & $33.2 \pm 1.6$ & $41.3 \pm 2.0$ & $33.4 \pm 1.4$ & $36.6 \pm 2.4$ \\
\hline Elongation (\%) & $37.2 \pm 1.2$ & $29.4 \pm 6.2$ & $20.9 \pm 3.2$ & $35.5 \pm 1.2$ & $39.4 \pm 6.4$ & $30.1 \pm 7.4$ \\
\hline
\end{tabular}

Bold values present the referring values of control CSfilms for modified films.

peak proving the Schiff-base formation between free amine groups and phenolic functional groups occurring near at $1650 \mathrm{~cm}^{-1}(\mathrm{C}=\mathrm{N}$ stretching mode of imines) was not clearly detected from our samples and it might be due to the reaction condition differences. The polymer products obtained by enzyme mediated processes are varied depending on the reaction conditions and Božič and his colleagues detected the products variety depending on $\mathrm{pH}$ condition and this peak was detected only at $\mathrm{pH} 5.5$ and $\mathrm{pH} 6.5$ conditions
$[11,13]$. However, the enzymatic synthesis was carried out at $\mathrm{pH}$ 5 in our study and showed a new peak appearance at $1610 \mathrm{~cm}^{-1}$ after reaction with catechin and laccase which might be occurred by Schiff-base formation between chitosan and polycatechin [36]. The NH stretching at $3300-3500 \mathrm{~cm}^{-1}$ become broader due to an increase of aromatic and non-aromatic stretching hydroxyl groups of phenols which result from a Michael addition reaction $[11,13,14]$. 

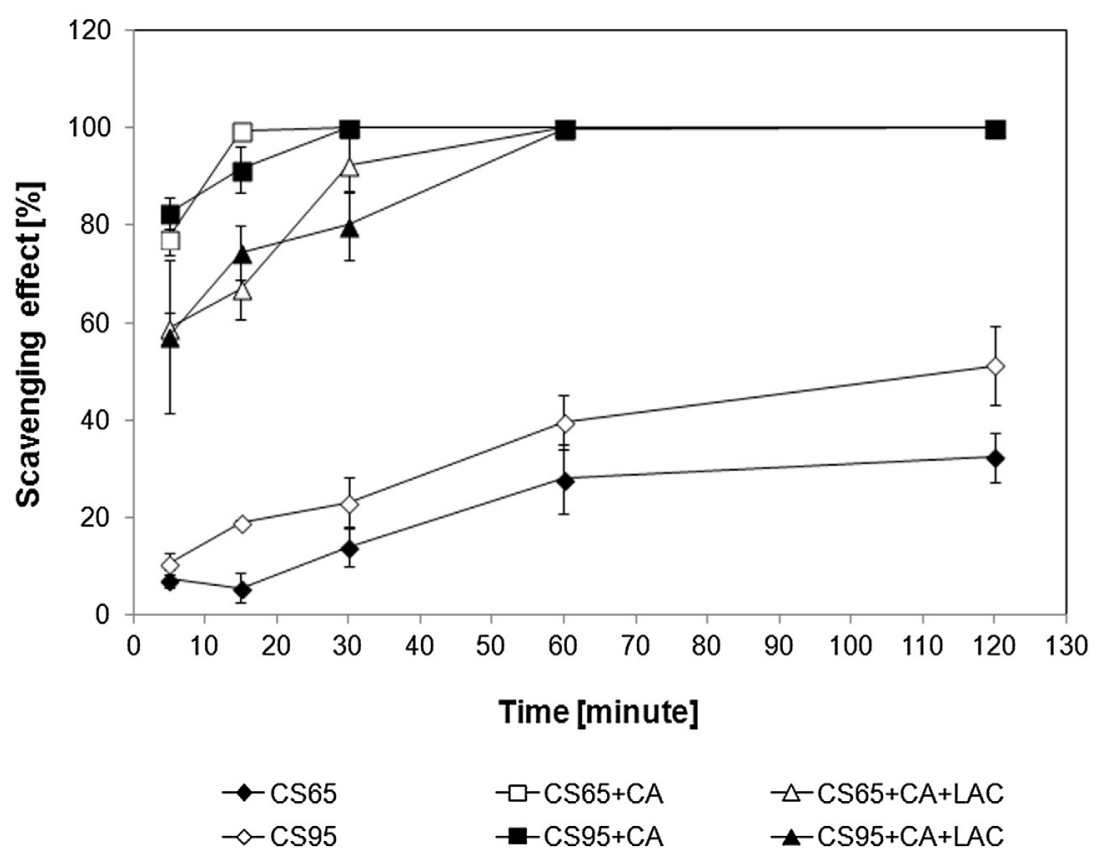

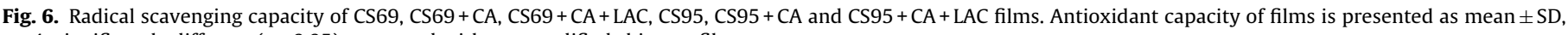
$\mathrm{n}=4$; significantly different $(p<0.05)$ compared with non modified chitosan films.

In wound dressing materials, the efficiency of fluid absorbance is an important factor to be considered to provide and maintain a moisturizing environment over the wound bed [43]. Moreover, swelling and erosion properties are important mechanisms to be considered as critical factors for drug release from hydrophilic matrices [42]. To study CS film behaviour in gastrointestinal environments, CS films were incubated in SIF and SGF separately at $37^{\circ} \mathrm{C}$ for $24 \mathrm{~h}$. The DDA, molecular weight, solvent $\mathrm{pH}$, types of acids, and thickness of films considerably affect the water permeability of the CS films $[44,45]$. The swelling capacity and erosion rate (weight loss) of each CS film in SIF for $24 \mathrm{~h}$ are presented in Table 3. The results showed no correspondence to the previous work of Motta de Moura and his colleagues that the lower molecular weight and higher DDA of CS have higher hydrophilicity and water vapour permeability [45]. Films prepared with CS69 showed a higher swelling rate than films prepared with CS95. CS69 and CS69+CA films absorbed the highest volume of water (EWu near $200 \%$ ). In the case the coupling reaction occurred between poly (CA)s and CS69 by enzymatic oxidation, the swelling rate decreased. Polymers produced by laccase mediation are highly insoluble [38] which might have affected on the level of water uptake. The films prepared with CS95 showed swelling rate values around 90 94\% which is less than half of swelling rate of CS65 films. This can be explained by the difference of the thickness of the films. All samples tested had a same size $\left(1.5 \times 1.5 \mathrm{~cm}^{2}\right)$ but had different initial weight and thickness (Table 3 ). CS95 had noticeably less weight and thickness, therefore, promoting less space for water retention capacity.

In the erosion test, the weight loss was measured before and after incubation for $24 \mathrm{~h}$, there was no significant weight loss detected, showing less than $3 \%$ of weight loss (Table 3) in all CS films. This might be due to $\mathrm{pH}$ dependence of $\mathrm{CS}$ ( $\mathrm{pKa}$ 6.3) and the semi-crystalline nature of CS which is derived mainly from inter and intra molecular hydrogen bonds [46]. Thus CS is hardly soluble in SIF ( $\mathrm{pH}$ 6.8) resulting in no notable weight change after incubation for $24 \mathrm{~h}$. On the contrary, CS films in SGF were rapidly dissolved and it was difficult to withdraw the films from the solution to be weighed $[46,47]$.
Continue with the swelling and erosion test, water permeability of CS films at certain time intervals is presented in Fig. 5. The films prepared with CS69 and CS95 show a slight difference in swelling behaviour over time. As chitosan is a pH sensitive compound, swelling behaviours of chitosan films at different buffer solutions were expected. Generally, the swelling rate of chitosan films was much higher in SGF (pH 1.2) than in SIF (pH 6.8) [47]. All CS films absorbed water rapidly in the first period in SIF (Fig. 5(A)). The swelling rate of CS69 and CS69+CA was higher than others and similar between them from two hours till $24 \mathrm{~h}$ of incubation time. The films prepared with CS95 showed three stages of swelling processes, they first increased, then decreased and finally became constant.

Weighing of CS films in SGF was very limited due to their high solubility and fast degradation in acidic solution ( $\mathrm{pH} 1.2$ ) (Fig. 5(B)). All CS films were decomposed and precipitated at the bottom of the test tube as a gel during the first stage of incubation ( $30 \mathrm{~min}$ ) except CS69 + CA + LAC and CS95 + CA + LAC films. The only sample that could be weighed without problems after $24 \mathrm{~h}$ of incubation was CS69 + CA + LAC. The swelling rate of CS95 + CA + LAC in SGF reached near $1400 \%$ in $30 \mathrm{~min}$, however, after $1 \mathrm{~h}$, the film decomposed and precipitated as other films.

Table 4 shows the ultimate tensile strength (UTS), and the elongation (\%) of CS films. CS69 film presented higher values of UTS, and elongation than CS95 film. In the literature, the DDA and MW were found to influence on the crystallinity and it can consequently affect on the mechanical properties of the chitosan films and the high UTS was obtained for high DDA CS than low DDA CS due to more active ionic sites in their molecules [45]. However, in our study, CS65 presented higher UTS than CS95 and it can be explained with the difference of thickness between CS65 and CS95. In Table 3, the CS65 was 1.5 times thicker than CS95, 47.5 and $31.5 \mu \mathrm{m}$, respectively and it might influence on the mechanical strength of films. The decrease of UTS was found in the CS films modified by enzymatic grafting with CA. The tensile strength of CS films was also affected by preparation conditions. The tensile strength of CS films decreases when they prepared with counter ions and the concentration of counter ion and hydroxyl groups are decisive factors for tensile strength of films [48]. It might be the reason of UTS decrease 

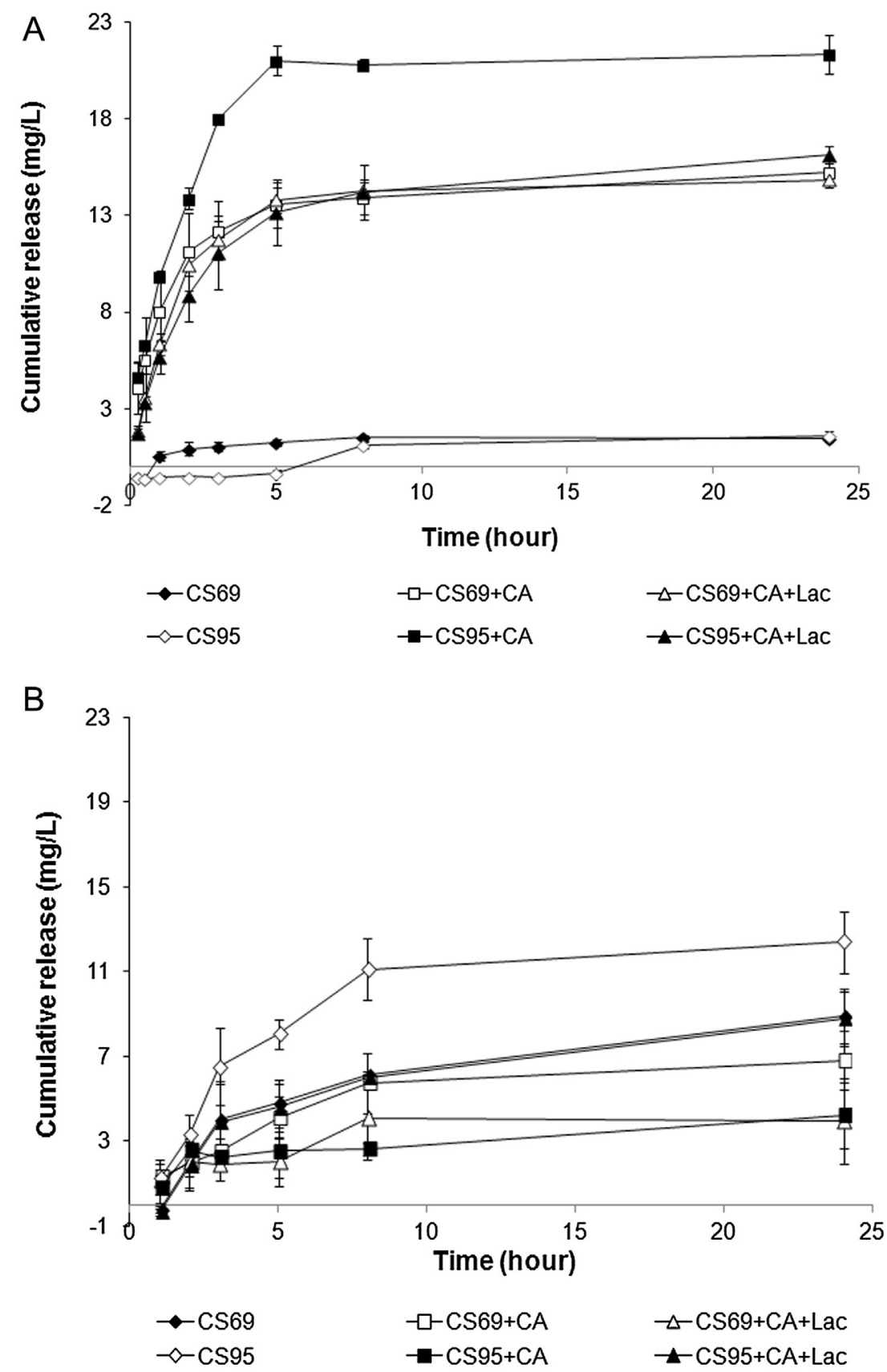

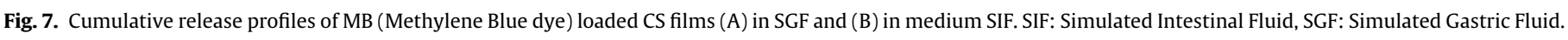

in CS films prepared with CA (CS + CA) which contains hydroxyl groups in the molecules. After occurring the enzymatic grafting of CA to CS, the slightly increased than CS + CA films but still lower than native CS films. The elongation (E) was not very affected by DDA and molecular weight of chitosan showing similar values [45] but affected by enzymatic grafting. Both CS65 and CS95 presented a decrease of elongation after modification and it might be same reason with UTS decrease.

ABTS•+ decolourisation assay method was applied to evaluate the antioxidant activities of CS films obtained by LAC mediated modification with CA. CS films prepared with CA in the presence and in the absence of LAC presented relatively strong scavenging activity against ABTS cation radicals (Fig. 6). The flavonoids are well known antioxidant agents with strong inhibition of autooxidation and scavenging of free radicals and have been broadly applied to the surface modification of materials to transfer the antioxidant activity $[10,11,13,31,37]$. The initial value of scavenging activity of CS95 + CA film was the highest but CS69 + CA film sample reached $100 \%$ of reduction of cation radical in shortest period of incubation time (in $15 \mathrm{~min}$ ). The laccase mediated polymerisation was identified by both an electrostatic interactions and additional ester-bond formation between the hydroxyl groups on C-6 CS and carboxyl group on flavonoid [11]. When phenolic acids in flavonoids formed oligomers or polymers by enzymatic oxidation, their antioxidant activity was reduced resulting similarly to monomers. This might be due to the low solubility and steric hindrance of the poly(CA)s $[11,38]$. The increase of antioxidant activity was observed in the films prepared with CA over time. Božič and his colleagues reported that this possibly occurred by the reproduction of reactive hydroxyl groups in the polymerised products, the high radical stability caused by greater electron delocalization and the structural characteristics of the oligomeric and polymeric products 

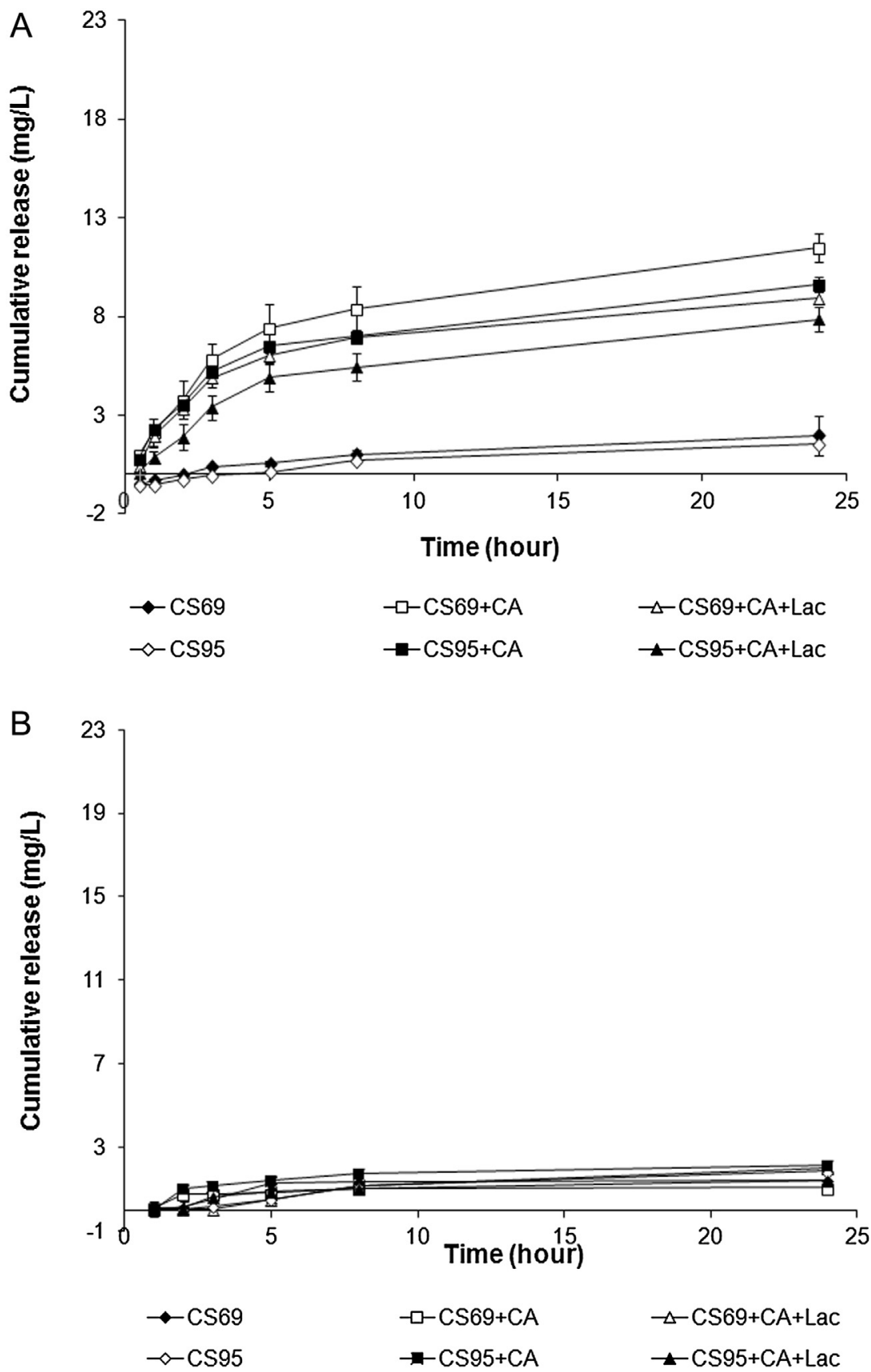

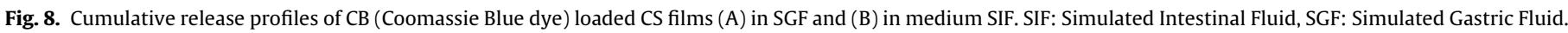

[11]. Although much lower than other samples the native CS films also showed some antioxidant activity. Chitosan has been studied as natural antioxidant and their scavenging activity was examined as radical scavengers against 1,1-diphenyl-2-picrylhydrazyl radicals (DPPH•), hydroxyl radical $\left(\bullet^{\bullet} \mathrm{OH}\right)$, and superoxide radical $\left(\bullet^{\bullet} \mathrm{O}_{2}-\right)$ $[49,50]$. There are several suggested mechanism explaining the chitosan scavenging the radicals and one of them is the elimination of free radicals by the action of nitrogen on the $\mathrm{C}-2$ position. The scavenging activity of chitosan derivatives against hydroxyl radicals may be derived from some or all the following: i) the hydroxyl groups in the polysaccharide unit can react with hydroxyl radicals by the typical $\mathrm{H}$-abstraction reaction $\mathrm{ii}$ ) $\mathrm{OH}$ can react with the residual free amino groups $\mathrm{NH}_{2}$ to form stable macromolecules radicals; iii) the $\mathrm{NH}_{2}$ groups can form ammonium groups $\mathrm{NH}_{3}{ }^{+}$by absorbing $\mathrm{H}^{+}$from the solution, then they react with $\mathrm{OH}$ through addition reactions $[37,50]$.
Natural biopolymers are becoming of greater interest for the controlled release of drugs. Chitosan is one of the most used natural polymers for medical and pharmaceutical applications for external and internal use, due to its properties like non-toxicity, antimicrobial activity and biocompatibility.

In this study, we have employed two different model compounds, MB and $\mathrm{CB}$, having different ionic charges. The chemical structure of drugs is very important to determine their release rates since their solubility and molecular weight are decisive parameters influencing their delivery by diffusion as well as the dissolution of the delivery matrix [42]. The release action of molecules from polymeric matrices may occur due to the mechanisms of Fick's diffusion, polymer matrix swelling, polymer matrix erosion and degradation [50,51]. The effect of the system environment on the release rate was monitored at different buffer media like SGF and SIF, and firstly described for MB molecules in CS films. During the washing pro- 
cess of the dried films, a high rate of colour loss occurred in MB loaded films, especially in native CS films. The colour loss might be related to the solubility of $\mathrm{MB}$ and the electrostatic interaction between $\mathrm{MB}$ and $\mathrm{CS}$. MB is highly water soluble at room temperature, compared to $\mathrm{CB}$, which is only slightly soluble in water therefore warming is needed to increase its solubility. With respect to the electrostatic charges, MB is positively charged in water and might have no charge attraction to bind to CS, which is either neutral or positively charged depending on $\mathrm{pH}$. From the obtained results shown in Fig. 7, the CA and LAC addition to the film is an important factor influencing on loading efficiency and release rate. The dye absorbance onto the films with CA or CA + LAC was much higher than unmodified CS69 and CS95 films, showing a stronger colouration. It was also observed that the MB loading delayed the degradation time in SGF medium showing an increase of release rate till $5 \mathrm{~h}$. The CS95 + CA film presented the highest release rate from the initial time, increasing to a constant value. Three other samples, CS69+CA, CS69 + CA + LAC and CS95 + CA + LAC, showed very similar release behaviours all throughout the same period of time. On the other hand, MB was poorly released in SIF medium ( $\mathrm{pH}$ 6.8 ) and continuously released without showing a constant value. This could be explained by the $\mathrm{pH}$ sensibility of CS which is hardly soluble at $\mathrm{pH} 6.8$. Even at $\mathrm{pH} 6.8,24 \%$ of amino groups of CS were still protonated [42] which explains the poor release of MB occurred in SIF medium. In both SGF and SIF media, the MB loaded native CS69 and CS95 films showed the lowest release rates.

The controlled release profiles of $\mathrm{CB}$ dye loaded CS films were also tested in SGF and SIF media and results obtained are presented in Fig. 8.

CB loaded CS films presented a stronger coloration but a lower release rate than MB loaded films. In this case, the strong electrostatic interaction between positively changed CS and negatively charged CB molecules increased the dye absorption onto films and prolonged the release time. In the reaction solution, the opposite charges of $\mathrm{CB}$ and $\mathrm{CS}$ form a strong ionic bond and thus decrease the $\mathrm{CB}$ release rate. Besides the charge interaction, the lower solubility and higher molecular weight of $\mathrm{CB}$ dye as compared to MB dye might influence on their release behaviours. In contrast to MB in CS films, CB was released faster from native CS films than CA grafted films, especially from CS95.

In SIF medium, the release rate of the dye was very low and it was difficult to differentiate the release profiles of the different CS films. The strong electrostatic interaction of CB with CS films together with the low solubility of chitosan in this medium might be responsible for this behaviour.

\section{Conclusions}

In the present study, laccase was used to catalyse catechin oxidation to produce reactive o-quinones and their subsequent reaction with free amino groups in chitosan through Schiff-base and Michael addition reactions. These reactions were proved by UV-vis and FT-IR spectroscopy which showed the characteristic peaks of the new functional groups. This functionalization was performed on chitosan with DDA of $69 \%$ and $95 \%$. The swelling, erosion, thermal and mechanical properties of functionalized CS films were studied. Antioxidant activity was highly improved when enzymatic grafting of CA occurred. The controlled release study of model compounds $\mathrm{MB}$ and $\mathrm{CB}$, showed that the DDA of CS, the type of loaded molecule, the release medium, and the enzymatic grafting of CA were important parameters that affected the release profiles of CS films.

\section{Declaration of interest}

The authors report no conflicts of interest. The authors alone are responsible for the content and writing of the paper.

\section{Acknowledgements}

This research was carried out by the Research Group of Materials Modification at Pontificia Universidad Católica del Perú PUCP. The authors would like to acknowledge to the support of Dirección de Gestión de la Investigación (DGI) and the Chemistry Department at Pontificia Universidad Católica del Perú PUCP for instrumental analysis.

\section{References}

[1] Z. Ahmadi, S. Oveisi, Z. Mohammadi Samani, Chitosan based hydrogels: characteristics and pharmaceutical applications, Res. Pharm. Sci. 10 (2015) $1-16$.

[2] R.A.A. Muzzarelli, J. Boudrant, D. Meyer, M. DeMarchis, M.G. Paoletti, Current views on fungal chitin/chitosan, human chitinases, food preservation, glucans, pectins and inulin: a tribute to Henri Braconnot, precursor of the carbohydrate polymers science, on the chitin bicentennial, Carbohydr. Polym. 87 (2012) 995-1012.

[3] S. Kim, J. Nakamatsu, D. Maurtua, F. Oliveira, Formation, antimicrobial activity, and controlled release from cotton fibers with deposited functional polymers, J. Appl. Polym. Sci. 133 (2016) 430-454.

[4] R.C.F. Cheung, T.B. Ng, J.H. Wong, W. Chan, Chitosan an update on potential biomedical and pharmaceutical applications, Mar. Drugs 13 (2015) 5156-5186.

[5] M. Mahdy Samar, M.H. El-Kalyoubi, M.M. Khalaf, M.M. Abd El-Razik, Physicochemical, functional, antioxidant and antibacterial properties of chitosan extracted from shrimp wastes by microwave technique, Ann. Agric. Sci. 58 (2013) 33-41

[6] N.A. Qinna, G.K. Qutuba, N. Al-Jbour, M.A. Al-Remawi, T.M. Alhussainy, Al. Al-So'ud KA, M.H. Omari, A.A. Badwan, Influence of molecular weight and degree of deacetylation of low molecular weight chitosan on the bioactivity of oral insulin preparations, Mar. Drugs 13 (2015) 1710-1725.

[7] I. Aranaz, R. Harris, I. Panos, B. Miralles, N. Acosta, G. Galed, A. Heras, Functional characterization of chitin and chitosan, Curr. Chem. Biol. 3 (2009) 203-230.

[8] M. Mittal, M.R. Siddiqui, K. Tran, S.P. Reddy, A.B. Malik, Reactive oxygen species in inflammation and tissue injury, Antioxid. Redox Signal. 20 (2014) $1126-1167$.

[9] S. Ghosh, S. Bhattacharyya, K. Rashid, P.C. Sil, Curcumin protects rat liver from streptozotocin-induced diabetic pathophysiology by counteracting reactive oxygen species and inhibiting the activation of p53 and MAPKs mediated stress response pathways, Toxicol. Rep. 2 (2015) 365-376.

[10] S. Kim, A. Cavaco-Paulo, Laccase-catalysed protein-flavonoid conjugates for flax fibre modification, Appl. Microbiol. Biotechnol. 93 (2012) 585-600.

[11] M. Božič, S. Gorgieva, V. Kokol, Laccase-mediated functionalization of chitosan by caffeic and gallic acids for modulating antioxidant and antimicrobial properties, Carbohydr. Polym. 87 (2012) 2388-2398.

[12] A.O. Aytekin, S. Morimura, K. Kida, Synthesis of chitosan-caffeic acid derivatives and evaluation of their antioxidant activities, J. Biosci. Bioeng. 111 (2011) 212-216

[13] M. Božič, Štrancar J, Kokol V, Laccase-initiated reaction between phenolic acids and chitosan, React. Funct. Polym. 73 (2013) 1377-1383.

[14] F. Sousa, G. Guebitz, V. Kokol, Antimicrobial and antioxidant properties of chitosan enzymaticallyfunctionalized with flavonoids, Process Biochem. 44 (2009) 749-756

[15] R. Shraddha Shekher, S. Sehgal, M. Kamthania, A. Kumar, Laccase: microbial sources, production, purification, and potential biotechnological applications, Enzyme Res. 2011 (2011) 1-11.

[16] M. Rodríguez-Delgado, G.S. Alemán-Nava, J.M. Rodríguez-Delgado, G. Dieck-Assad, S.O. Martínez-Chapa, D. Barcelóc, R. Parra, Laccase-based biosensors for detection of phenolic compounds, Trends Anal. Chem. 74 (2015) 21-45.

[17] P. Upadhyay, R. Shrivastava, P.K. Agrawal, Review: bioprospecting and biotechnological applications of fungal laccase, 3, Biotech 6 (2015) 1-12

[18] D.P. Chattopadhyay, M.S. Inamdar, Aqueous behaviour of chitosan, Int. J. Polym. Sci. 2010 (2010) 1-7.

[19] Y.S. Puvvada, S. Vankayalapati, S. Sukhavasi, Extraction of chitin from chitosan from exoskeleton of shrimp for application in the pharmaceutical industry, Int. Curr. Pharm. J. 1 (2012) 258-263.

[20] W. Wang, S. Bo, S. Li, W. Qin, Determination of the Mark-Houwink equation for chitosans with different degrees of deacetylation, Int. J. Biol. Macromol. 1 (1991) 281-285.

[21] M. Kasaai, J. Arul, G. Charlet, Intrinsic viscosity-molecular weight relationship for chitosan, J. Polym. Sci. B Polym. Phys. 38 (2014) 2591-2598. 
[22] R. Pamies, J.G.H. Cifre, M.C. Martínez, Torre JG. Determination of intrinsic viscosities of macromolecules and nanoparticles. Comparison of single-point and dilution procedures, Colloid. Polym. Sci. 286 (2008) 1223-1231.

[23] E. Fernandez-Megia, R. Novoa-Carballal, E. Quiñoá, R. Riguera, Optimal routine conditions for the determination of the degree of acetylation of chitosan by 1H-NMR, Carbohydrate Polymers, Carbohydr. Polym. 61 (2005) 155-161.

[24] M. Lavertu, Z. Xia, A.N. Serreqi, M. Berrada, A. Rodrigues, D. Wang, A validated $1 \mathrm{H}$ NMR method for the determination of the degree of deacetylation of chitosan, J. Pharm. Biomed. Anal. 32 (2003) 1149-1158.

[25] V. Singh, K. Kumari, Synthesis and characterization of chitosan-starch crosslinked film for controlled drug release, Int. J. Mater. Biomater. Appl. 4 (2014) 7-13.

[26] A. Thakur, S. Monga, R.K. Wanchoo, Sorption and 581 drug release studies from semi-interpenetrating polymer networks of chitosan and xanthan gum, Chem. Biochem. Eng. Q. 28 (2014) 105-115.

[27] L. Liang, L. Jinfengm, S. Shanshan, W. Linlin, S. Chenjun, S. Yujiao, L. Zhenglin, M. Shirui, Effect of formulation variables on in vitro release of a water-soluble drug from chitosan-sodium alginate matrix tablets, Asian J. Pharm. 10 (2015) $314-321$.

[28] R. Re, N. Pellegrini, A. Proteggente, A. Pannala, M. Yang, C. Rice-Evans, Antioxidant activity applying an improved ABTS radical cation decolorization assay, Free Radic. Biol. Med. 26 (1999) 1231-1237.

[29] M. Hussain, M. Iman, T.K. Maji, Determination of degree of deacetylation of chitosan and their effect on the release behavior of essential oil from chitosan and chitosangelatin complex microcapsules, Int. J. Adv. Eng. Appl. 2 (2013) 4-12.

[30] S. Arabi, M.R. Sohrabi, Removal of methylene blue, a basic dye, from aqueous solutions using nano-zerovalent iron, Water Sci. Technol. 70 (2014) 24-31.

[31] P.R. Ginimuge, S.D. Jyothi, Methylene blue: revisited, J. Anaesthesiol. Clin. Pharmacol. 26 (2010) 517-520.

[32] X.X. Han, L. Chen, J. Guo, B. Zhao, Coomassie brilliant dyes as surface-Enhanced raman scattering probes for protein-Ligand recognitions, Anal. Chem. 82 (2010) 4102-4106.

[33] T. Heck, G. Faccio, M. Richter, L. Thöny-Meyer, Enzyme-catalyzed protein crosslinking, Appl. Microbiol. Biotechnol. 97 (2013) 461-475.

[34] R.N. Tharanathan, F. Kittur, Chitin-the undisputed biomolecule of great potential, Crit. Rev. Food Sci. 43 (2003) 61-87.

[35] Y. Yuan, M.C. Betsy, O. Warren, J.D. Bumgardner, Deacetylation of chitosan: material characterization and in vitro evaluation via albumin adsorption and pre-osteoblastic cell cultures, Materials 4 (2011) 1399-1416.

[36] A. Aljawish, I. Chevalot, B. Piffaut, C. Rondeau-Mouro, M. Girardin, J. Jasniewski, J. Scher, Functionalization of chitosan by laccase-catalyzed oxidation of ferulic acid and ethyl ferulate under heterogeneous reaction conditions, Carbohydr. Polym. 87 (2012) 537-544.

[37] C. Silva, T. Matamá, S. Kim, J. Padrão, E. Nugroho, T. Kudanga, G. Nyanhongo, G. Guebitz, M. Casal, A. Cavaco-Paulo, Antimicrobial and antioxidant linen via laccase-assisted grafting, React. Funct. Polym. 71 (2011) 713-720.
[38] L. Gianfreda, F. Sannino, M.A. Raoa, J.M. Bollag, Oxidative transformation of phenols in aqueous mixtures, Water Res. 37 (2003) 3205-3215.

[39] A.M. Osman, K.K.Y. Wong, A. Fernyhough, The laccase/ABTS system oxidizes (+)catechin to oligomeric products, Enzyme Microb. Technol. 40 (2007) 1272-1279.

[40] L. Li, L. Wang, Y. Shao, R. Ni, T. Zhang, S. Mao, Drug release characteristics from chitosan-alginate matrix tablets based on the theory of self-assembled film, Int. J. Pharm. 450 (2013) 197-207.

[41] Y. Youling, B.M. Chesnutt, W.O. Haggard, J.D. Bumgardner, Deacetylation of chitosan: material characterization and in vitro evaluation via Albumin adsorption and pre-osteoblastic cell cultures, Materials 4 (2011) 1399-1416.

[42] K. Kim, S.K. Kim, C.L. Weller, M.A. Hanna, Properties of chitosan films as a function of pH and solvent type, J. Food Sci. 71 (2006) 119-124.

[43] L.J.R. Foster, S. Ho, J. Hook, M. Basuki, H. Marçal, Chitosan as a biomaterial: influence of degree of deacetylation on its physiochemical, material and biological properties, PLoS One 10 (2015) 1-22.

[44] C. Motta de Moura, J. Motta de Moura, N.M. Soares, L.A. Pinto, Evaluation of molar weight and deacetylation degree of chitosan during chitin deacetylation reaction: used to produce biofilm, Chem. Eng. Process. 50 (2011) 351-355.

[45] A. Doulabi, H. Mirzadeh, M. Imani, N. Samadi, Chitosan/polyethylene glycol fumarate blend film: physical and antibacterial properties, Carbohydr. Polym. 92 (2013) 48-56.

[46] I. Sogias, V. Khutoryanskiy, A.C. Williams, Exploring the factors affecting the solubility of chitosan in water, Macromol. Chem. Phys. 211 (2010) 426-433.

[47] X.Z. Shu, K.J. Zhu, W. Song, Novel pH-sensitive citrate cross-linked chitosan film for drug controlled release, Int. J. Pharm. 212 (2001) 19-28.

[48] A. Rajalakshmi, N. Krithiga, A. Jayachitra, Antioxidant activity of the chitosan extracted from shrimp exoskeleton middle, East J. Sci. Res. 16 (2013) 1446-1451.

[49] H. Li, Q. Xu, Y. Chen, A. Wan, Effect of concentration and molecular weight of chitosan and its derivative on the free radical scavenging ability, J. Biomed. Mater. Res. A 102 (2013) 911-916.

[50] N. Faisant, J. Siepmann, J.P. Benoit, PLGA-based microparticles: elucidation of mechanisms and a new, simple mathematical model quantifying drug release, Eur. J. Pharm. Sci. 15 (2002) 355-366.

[51] D. Klose, F. Siepmann, J.F. Willart, M. Descamps, J. Siepmann, Drug release from PLGA-based microparticles: effects of the microparticle:bulk fluid ratio, Int. J. Pharm. 383 (2010) 123-131. 